included a detailed medical history, psychometric testing, a battery of cognitive tests and EEG recordings in response to cognitive and emotion-related tasks. A new measure of neural synchrony was used to quantify phase synchrony within EEG bands, focusing on gamma.

Results: FES was defined by marked impairments in social functioning and perceived quality of life, related to severity of negative symptoms. This clinical profile was associated with similarly marked deficits on cognitive measures of executive function, working memory and emotion perception. In terms of neural synchrony, a pattern of 'hypersynchrony' compared with healthy controls was apparent in response to processing of salient information.

Conclusions: These findings provide support for a model of FES that focuses on alterations in synchronization of brain function required for effective binding of complex and significant stimuli. Because poor emotional function and negative symptoms in FES are valid predictors of 'real-world' functional outcome, neural synchrony markers show promise as an objective marker of illness progression.

\section{Happy trails to you - a review of subjective well-being in successful aging}

\author{
A Withall ${ }^{1,2}$, H Brodaty ${ }^{1}$ \\ 'Prince of Wales Hospital/University of New South Wales; and \\ ${ }^{2}$ The University of Sydney, Sydney, Australia
}

Background: Many studies have examined depression in aging with the assumption that this also provides information concerning the nature of happiness. There is consequently a paucity of research on happiness, in particular its determinants and relationship with successful aging. The aim of this research was to review studies concerning happiness in aging.

Methods: MEDLINE, PsychINFO, CINAHL and EBM reviews were searched from their inception to June 2006, using the terms happiness, optimism, positive affect, subjective well-being, well-being and life satisfaction.

Results: The main findings can be summarized as follows: most studies support an increase in happiness with age although some longitudinal research has shown decreases over the life span. Correlates of happiness and subjective well-being in aging include marital status, religious commitment, subjective ratings of health status, social capital, task- or avoidance-oriented coping, cognitive function and housing quality. Positive affect appears as much of a protective factor as negative affect is a risk factor for functional disability in aging, especially following illness or injury. Finally, early results suggest that positive psychology interventions such as the 'good-things-in-life' exercise may be efficacious in increasing and maintaining levels of happiness.

Conclusion: Happiness is an important contributor to successful aging and further intervention studies should be pursued to improve the health of older persons.

\section{Behavioural problems following stroke - is there a relationship with cognitive impairment?}

\section{A Withall ${ }^{1,2}$, H Brodaty ${ }^{1}$, A Altendorf', P Sachdev'}

'Prince of Wales Hospital/University of New South Wales; and

${ }^{2}$ The University of Sydney, Sydney, Australia

Background: This study aimed to show the contribution of cognitive impairment toward the development of behavioural disturbance following stroke.

Method: Subjects in the Sydney Stroke Study comprised patients admitted to two university hospitals after an ischemic stroke and controls from the community who received extensive medical, psychiatric and neuropsychological assessments, with a subset receiving a magnetic resonance imaging scan. Patients were assessed 3-6 months after their stroke and again a year later. Controls were similarly assessed twice, 12 months apart. This sample comprised 123 stroke patients and 88 control subjects, with complete ratings on cognitive impairment at 15 months.

Results: Of the 88 controls, $55.7 \%$ were classified as having no cognitive impairment, $30.7 \%$ had mild cognitive impairment and $2.3 \%$ had dementia at 15 months. Of the 123 patients, $42.3 \%$ had no cognitive impairment, $39.8 \%$ had VCI and $16.3 \%$ had dementia. The stroke group had significantly higher rates of dementia [odds ratio (OR): $8.35,95 \%$ confidence interval (CI): 1.90-36.73] but not of cognitive impairment (OR: $1.71,95 \%$ CI: 0.93-3.15). Using nonparametric correlation, total NPI score was correlated with cognitive impairment in the total sample (Spearman's $\rho=0.27, P=0.001)$. Within the stroke group, dementia was significantly associated with NPI score at 15 months but not MMSE, stroke severity, IADL/ ADL score, more than one stroke, total stroke volume, total atrophy or total white matter hyperintensities.

Conclusion: Having a stroke does not necessarily lead to behavioural disturbance; however, it is associated with higher rates of cognitive impairment (in particular dementia), which in turn is associated with more disturbance. 